\title{
Effects of plasticization of a soft silicone for dielectric elastomer actuation
}

\author{
Fabia Galantini $^{* *}$, Federico Carpi ${ }^{2}$, Giuseppe Gallone ${ }^{1,3}$ \\ ${ }^{1}$ Research Centre "E. Piaggio", University of Pisa, Largo Lucio Lazzarino 2, 56122, Pisa, Italy. \\ ${ }^{2}$ School of Engineering \& Materials Science, Queen Mary University of London, Mile End Road, \\ London E1 4NS, UK \\ ${ }^{3}$ Department of Civil and Industrial Engineering, Largo Lucio Lazzarino 2, 56122, Pisa, Italy \\ *Contact author. E-mail: fabia.galantini@,ing.unipi.it
}

\begin{abstract}
Dielectric elastomer (DE) actuators exploit electrically induced deformations of insulating rubbery materials, as a means to transduce electrical energy into mechanical work. To enable large deformations, recent studies have demonstrated the advantage of either using elastomers that suppress pull-in electromechanical instability or driving the actuator at the verge of instability while still preventing it. Whenever these strategies are not applicable, softening the material remains the mechanical approach of choice to enable large deformations at relatively low electric fields. As the most common approach to lower the elastic modulus of an elastomer is the use of plasticizers, understanding their effects also on other properties of the elastomer is important, especially to actuator designers. Aimed at gaining insights in this respect, this paper presents an extensive chemical-physical, dielectric, mechanical and electromechanical characterisation, for different amounts of a plasticizer, of one of the softest commercial silicones demonstrated for DE actuation (elastic modulus of the order of $100 \mathrm{kPa}$ ). The results showed the interplaying effects of a variable addition of the plasticiser, elucidating key features that could thus serve as a guide to the design of actuators for specific needs.
\end{abstract}

Keywords: Actuator, dielectric elastomer, EAP, electroactive, plasticizer, polymer, silicone, soft.

\section{Introduction}

Several means for transducing electrical energy into mechanical work are currently available in order to build electromechanical actuators. Among them, devices based on electro-responsive polymeric materials, known as electroactive polymers or electromechanically active polymers (EAPs), are undergoing considerable development [1]. In particular, dielectric elastomers (DEs) are progressively emerging as one of the most performing classes of EAP materials [2-5]. Providing a DE film with compliant electrodes makes the whole structure to undergo deformation upon electrical charging. Indeed, the oppositely charged electrodes attract each other electrostatically, giving rise to a compression of the elastomer, sustained by the following effective pressure [2]:

$p=\varepsilon_{0} \varepsilon_{r} E^{2}$

where $\varepsilon_{0}$ is the dielectric constant of vacuum $\left(\varepsilon_{0}=8.85 \times 10^{-12} \mathrm{~F} / \mathrm{m}\right), \varepsilon_{r}$ is the relative dielectric constant of the elastomer and $E$ is the applied electric field. The resulting output mechanical work can be exploited for actuation.

The compliance of the elastomer along with its dielectric strength enable such a simple electromechanical transduction mechanism. The softer the material is, the lower the necessary driving electric field is, in order to get a given strain. 
However, a high compliance is not always desirable. Indeed, one should take into account that this is usually accompanied by low achievable forces. Nevertheless, maximizing the output force is not always a primary aim. In fact, many applications frequently require high displacements at low forces. This is the case, for instance, of micro-mechanisms to stretch biological cells in vitro [6], or tuneable optical systems, such as diffractive gratings [7] and variable-focus lenses [8].

In order to enable large deformations of DE structures upon electrical driving, recent studies have demonstrated the advantage of either using elastomers that suppress pull-in electromechanical instability [9] or driving the actuator at the verge of instability while still preventing it [10]. Whenever these advanced strategies are not applicable, the increase of the material's compliance (i.e. the reduction of its elastic modulus) remains the mechanical approach of choice to enable large deformations at relatively low electric fields.

The most common solution to lower the elastic modulus of an elastomer is the use of plasticizers. Therefore, understanding the effects of these material components also on other properties of the elastomer is important, especially to actuator designers. This paper focuses on this aspect, and presents an extensive characterisation, for different amounts of a plasticizer, of one of the softest commercial silicones demonstrated for DE actuation. The elastomer was used in the past, both 'as it is', to demonstrate new DE actuators, such as helical [11], folded [12], bucking [13] and hydrostatically coupled [14] devices, and to develop new DE materials as composites and blends [15-18]. Despite this, no wide assessment of the dependence on the plasticiser's content of the material's chemical-physical, dielectric, mechanical and electromechanical properties that underpin its use for DE actuation has ever been reported so far, thus motivating the work presented here.

\section{Materials and methods}

\subsection{Material processing}

The characterised material is a silicone elastomer (TC-5005 A/B-C, BJB Enterprises Inc., USA), available as a three components product: a pre-polymer (A), a curing agent (B) and a plasticizer $(\mathrm{C})$. A base rubber is obtained by mixing the curing agent with the pre-polymer at a ratio of 10 parts by weight of B per 100 of A. As declared by the manufacturer, to soften the resulting material, the plasticizer $\mathrm{C}$ can be added with a maximum content of $50 \mathrm{phr}$ (parts per hundred rubber). In this study, the variation of the material properties with the amount of softener was investigated by producing samples at plasticizer contents of $0,10,20,40$ and $45 \mathrm{phr}$. In all cases, each formulation was cured in aluminium moulds at room temperature for one day, obtaining silicone films with a thickness of about $1 \mathrm{~mm}$.

For comparison purposes, electromechanical tests were also carried out on samples of one of the most studied material for DE actuation: the VHB 4905 acrylic elastomer film supplied by $3 \mathrm{M}$.

\subsection{IR and NMR spectroscopy}

According to the manufacturer, the silicone material under study consists of a poly(dimethyl siloxane) mixture. No additional information is available about the chemical composition of its components (pre-polymer, curing agent and plasticizer). To achieve more insights, both infra-red (IR) and nuclear magnetic resonance (NMR) spectroscopy were carried out on the three reagents. In particular, Fourier Transform IR (FT-IR) transmission spectra were acquired at $20^{\circ} \mathrm{C}$ by using a Perkin-Elmer Spectrum One spectrometer. Hydrogen-NMR analysis was carried out at $20^{\circ} \mathrm{C}$ by means of a Varian Gemini $200 \mathrm{MHz}$ spectrometer on samples dissolved in $5-10 \% \mathrm{CDCl}_{3}$ solutions (Aldrich, 99.8 D atom \%).

\subsection{Dielectric spectroscopy}

Dielectric spectroscopy was carried out at $20^{\circ} \mathrm{C}$ on samples at various plasticizer contents. The procedure described in [16] was used to assess the frequency dependence, in the range 10-10 $\mathrm{Hz}$, of both the real and imaginary parts of the relative complex dielectric permittivity: 


$$
\varepsilon_{r}^{*}(\omega)=\varepsilon_{r}{ }^{\prime}(\omega)-j \varepsilon_{r}{ }^{\prime \prime}(\omega)
$$

where $\omega$ is the angular frequency. Tested specimens were shaped as discs, having a thickness lower than $1 \mathrm{~mm}$ and a diameter of $11 \mathrm{~mm}$.

\subsection{Mechanical tests}

Uniaxial tensile quasi-static tests and dynamic-mechanical analysis (DMA) were performed on the set of elastomer samples, as described below.

The quasi-static measurements were carried out on specimens shaped as rectangular stripes of about $1 \mathrm{~mm} \times 5 \mathrm{~mm} \times 50 \mathrm{~mm}$, by applying step-wise increasing loads and by measuring the correspondent elongations. Each elongation measurement was taken after three minutes from each application of a new stress value.

In order to study the viscoelastic properties of the material and to assess the dependence of its complex elastic modulus

$$
Y^{i}(\omega, T)=Y^{\prime}(\omega, T)+j Y^{\prime \prime}(\omega, T)
$$

on the frequency $\omega$ and temperature $T$, both temperature (DMTA) and frequency (DMA) sweep analyses were performed by using a dynamic testing instrument (Eplexor 100N, Gabo Qualimeter Testanlagen GMBH, Germany). The temperature behaviour was studied in the range from -100 to $50{ }^{\circ} \mathrm{C}$ at a fixed frequency of $100 \mathrm{~Hz}$, while the frequency analysis was carried out in the range from 0.1 to $100 \mathrm{~Hz}$ at a fixed temperature of $22{ }^{\circ} \mathrm{C}$. For both measurements, the applied mechanical stimulus consisted of a bias static strain of $5 \%$ with a superimposed dynamical strain of $1 \%$.

\subsection{Electromechanical tests}

The actuation performance of both the processed materials and the VHB 4905 acrylic elastomer was investigated by assembling elementary planar actuators, as follows. The samples were shaped as rectangular stripes with sizes varying from 35 to $65 \mathrm{~mm}$ in length and from 16 to 18 $\mathrm{mm}$ in width, and with a thickness of about $1 \mathrm{~mm}$ for the silicone samples, while only for the VHB 4905 sample the thickmess was $0.5 \mathrm{~mm}$, i.e. that of the film as supplied by the manufacturer. Thereafter, the opposite ends of each stripe were glued to stiff plastic supports (Fig. 1). Compliant electrodes were created on the two main surfaces of each stripe, by smearing carbon conductive grease (Nyogel 755G, Tecnolube Seal, U.S.A.).

The electromechanical isotonic strain of each actuator, arising in response to different applied electric fields, was measured along a transverse (orthogonal) direction with respect to the field. For this purpose, each actuator was arranged in a vertical position, by clamping its lower end and connecting the higher one to an isotonic displacement transducer (7006, Ugo Basile, Italy), as shown in Fig. 1. The transducer was used not only to measure the electrically induced vertical strain, but also to apply a passive vertical pre-strain of $100 \%$. This value was chosen according to the outcomes of uniaxial tensile tests, reported in the next section. Indeed, a strain of $100 \%$ is roughly representative (same order of magnitude) of the strain level around which the stress-strain curves of the tested materials showed an oblique flex point, i.e. the point at which the material holds the minimum tangent elastic modulus (minimum slope) and so it exhibits the maximum variation of strain for any given variation of stress. For this reason, such a bias strain was applied to maximize the material deformation in response to any applied electric field.

In order to quantify the electric field arising from any applied voltage, the thickness of each pre-strained sample was estimated as follows. By assuming an incompressibility of the material, the occurring thickness pre-strain $S_{z z, p}$ was calculated from the imposed vertical pre-strain $S_{x x, p}=100 \%$, by means of the following relation [19]:

$$
S_{z z, p}=-1+1 / \sqrt{1+S_{x x, p}}
$$


where the Cartesian axes $x, y, z$ are defined in Fig. 1. It is worth remarking that Eq. (4) holds for a sample of elastomer unconstrained on the $y z$ plane and for a strain symmetry along the axes $y$ and $z$ $\left(S_{y y, p}=S_{z z, p}\right)$. These hypotheses were assumed to be approximately satisfied by the considered experimental set-up. Indeed, although the two plastic supports introduced a constraints along the direction $y$ (Fig. 1), any portion of the specimen opportunely far from these constrained ends (i.e. any central part) was assumed to be capable of free deformation along $x, y$ and $z$. Eq. (4) returned $S_{z z, p} \cong-29.3 \%$, which means that the elastomer film thickness changed, upon pre-stretching, from an unstrained value $z_{u} \cong 1 \mathrm{~mm}$ to a pre-strained value $z_{0} \cong 0.7 \mathrm{~mm}$, according to the following expression:

$z_{0}=z_{u}\left(1+S_{z z, p}\right)$

This value was then used to estimate the applied nominal electric field (initial value) as the ratio between the applied voltage and $z_{0}$.

Active strains were then induced in pre-strained actuators by applying step-wise increasing voltages, generated by a dc high-voltage power supply (HV-DC 205A-30P, Bertan, U.S.A.), till occurrence of the material's dielectric breakdown. For each voltage value, the steady-state transverse strain was recorded along the vertical $(x)$ direction (Fig. 1).

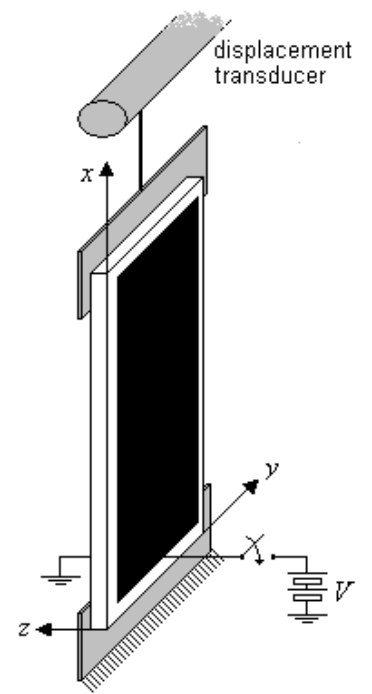

Figure 1. Schematic drawing of the experimental set-up for the electromechanical tests.

\section{Results and discussion}

Figs. 2 and 3 present the IR and NMR characterisation results, showing that in each case the spectra of the material components $(\mathrm{A}, \mathrm{B}, \mathrm{C})$ are very similar to each other.

In particular, concerning the IR analysis (Fig. 2), main absorption peaks are located at 3000, $1260,1000-1100$ and $800 \mathrm{~cm}^{-1}$. Secondary peaks are also observable at 1900,1400 and $700 \mathrm{~cm}^{-1}$ for all the three components. The similarity of the IR spectra is indicative of a similar molecular structure of the three components. Specifically, the main peaks are characteristic of well-known phenomena: the $\mathrm{C}-\mathrm{H}$ stretching (peak at $3000 \mathrm{~cm}^{-1}$ ), the $\mathrm{Si}-\mathrm{CH}_{3}$ and $\mathrm{Si}\left(\mathrm{CH}_{3}\right)_{2}$ stretching (peaks at $1260 \mathrm{~cm}^{-1}$ and $800 \mathrm{~cm}^{-1}$ ) and the Si-O-Si vibration (peak around $1000 \mathrm{~cm}^{-1}$ ). The double splitting of the latter peak is a typical feature of the siloxane group, particularly evident for long-chains molecules. Secondary peaks could likely be attributed to phenilic groups, although however a reliable interpretation is difficult. In fact, siloxanes typically show great absorptions even for little concentrations of material and their spectra can hide possible additives, even for significant amounts. 


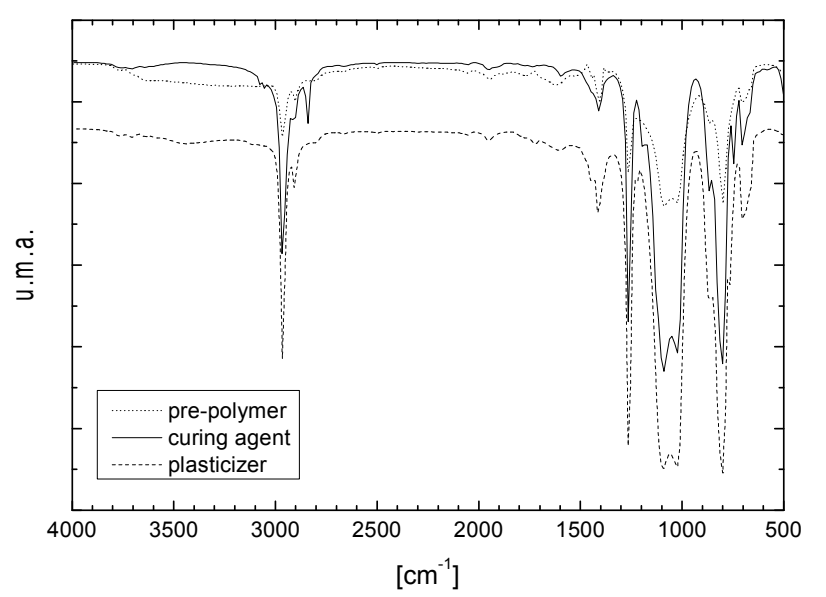

Figure 2. IR spectra of pre-polymer, curing agent and plasticizer.

Concerning the NMR analysis (Fig. 3), the pre-polymer spectrum shows only two absorption peaks, one at $7.26 \mathrm{ppm}$, typical of $\mathrm{CDCl}_{3}$ solvent, and the second around $0 \mathrm{ppm}$, due to $\mathrm{Si}^{-\mathrm{CH}_{3}}$ groups. For the spectrum of the curing agent, main absorption peaks are located at 7.60, 7.26, 3.63 and $0 \mathrm{ppm}$, which can respectively be attributed to $-\mathrm{PhH}, \mathrm{CDCl}_{3},-\mathrm{OCH}_{3}$ and $\mathrm{Si}_{-}-\mathrm{CH}_{3}$ groups. However, other not specified weak absorptions are also visible at 3.49, 1.55 and $0.95 \mathrm{ppm}$, indicating that the curing agent contains a variety of functional groups with respect to the prepolymer. As for the IR analysis, the NMR spectrum of the plasticizer results very similar to that of the pre-polymer, meaning that they share almost the same chemical nature and should mainly differ just for the molecular weight. Therefore, IR and NMR spectra of these components as a whole are consistent with the poly(dimethyl-siloxane) nature of the material.

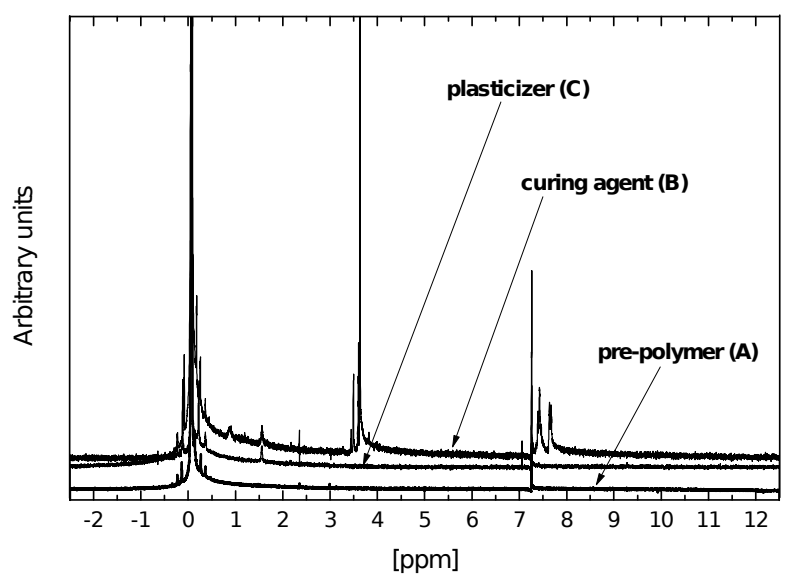

Figure 3. NMR spectra of pre-polymer, curing agent and plasticizer.

The similarity of the chemical composition of the material components is reflected also by the dielectric spectra of the set of elastomers, presented in Fig. 4. For each sample, both the dielectric constant and loss properties show a very smooth and slightly dispersive behaviour. In particular, values of $\varepsilon_{r}{ }^{\prime}$ and $\varepsilon_{r}{ }^{\prime \prime}$ at all frequencies are included approximately in the ranges 4.0-5.5 and $0-0.7$, respectively. Concerning the effect of the plasticizer addition, the $\varepsilon_{r}{ }^{\prime}$ values show a slight monotonic increase with the plasticizer content at frequencies above $10 \mathrm{kHz}$, i.e. where the orientation polarization processes dominate the dielectric response and the spectrum is found to be almost flat. Below $10 \mathrm{kHz}$, instead, all the spectra of the various formulations show slightly varying low frequency dispersion trends and intersect each other, however without any recognizable regularity with the plasticizer content. The $\varepsilon_{r}{ }^{\prime \prime}$ spectra of the samples appear to be almost 
completely unaffected by the plasticizer content at high frequencies, while at lower frequencies they start to separate each other but remaining tightly and, again, irregularly distributed around the $0 \mathrm{phr}$ sample spectrum. Indeed, both the real and imaginary parts of the relative permittivity reveal to be scarcely affected by the content of plasticizer, and all the systems behave as very low polarizable and very low dissipative dielectrics.
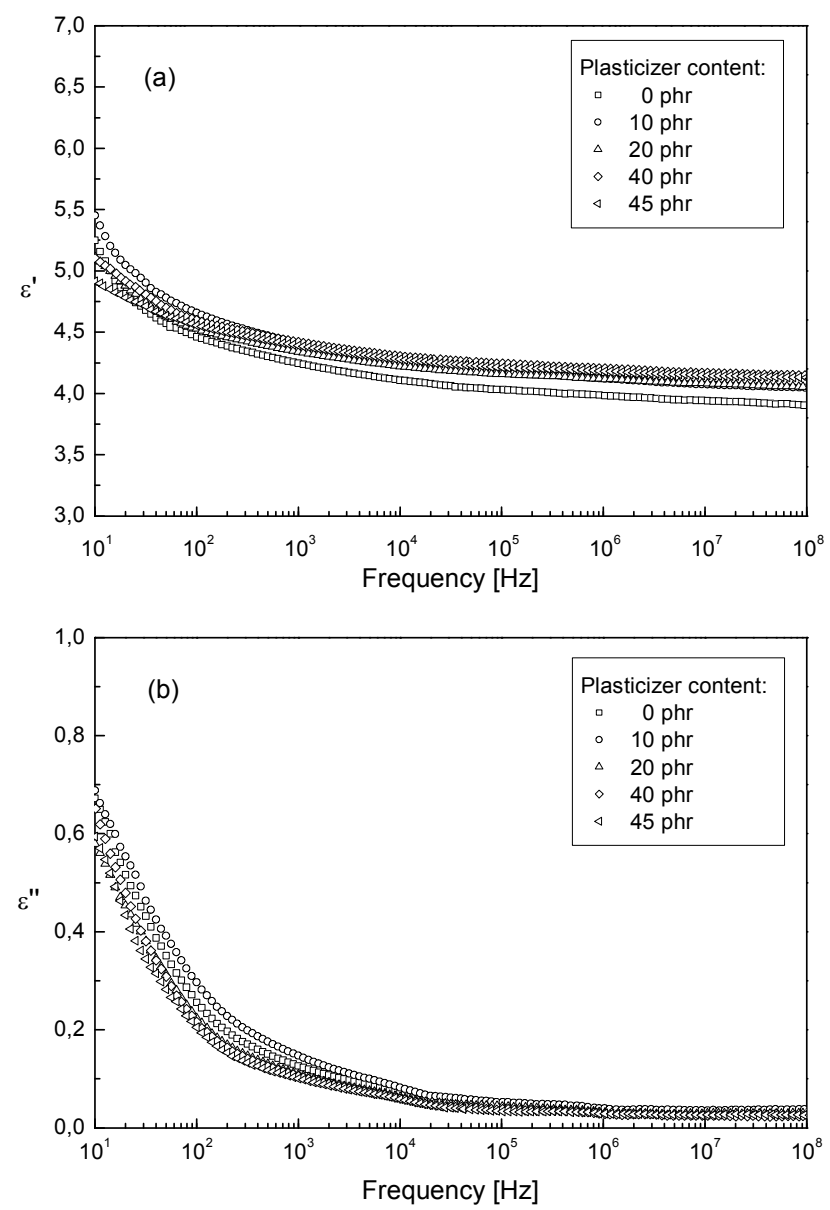

Figure 4. Dielectric spectra of the elastomers at different plasticizer contents: (a) real part; (b) imaginary part.

While the plasticizer did not show any significant effect on the dielectric permittivity, a different behaviour was observed for the mechanical properties, as expected. The stress-strain curves reported in Fig. 5 show that an increase of the amount of plasticizer (which was found to have a chemical structure similar to that of the pre-polymer but lower molecular weight) corresponds to a monotonic reduction of the material's elastic modulus, down to about 5 times at low strains, as pointed out in Fig. 6. The plasticizer content also influences both the elongation at break, whose values raise from about $230 \%$ for the base rubber (neat A/B mixture) up to more than $400 \%$ for the sample containing $45 \mathrm{phr}$ of plasticizer, and the stress at break, which drops from about $300 \mathrm{kPa}$, for the base rubber, down to $150 \mathrm{kPa}$ for the elastomer with the maximum content of C. 


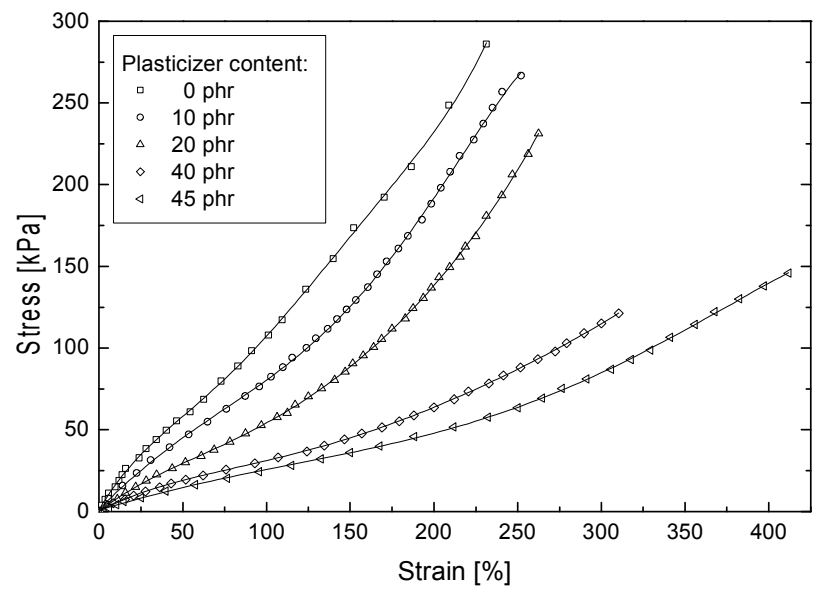

Figure 5. Stress-strain curves of the elastomers at different plasticizer contents (lines are just guides for the eye).

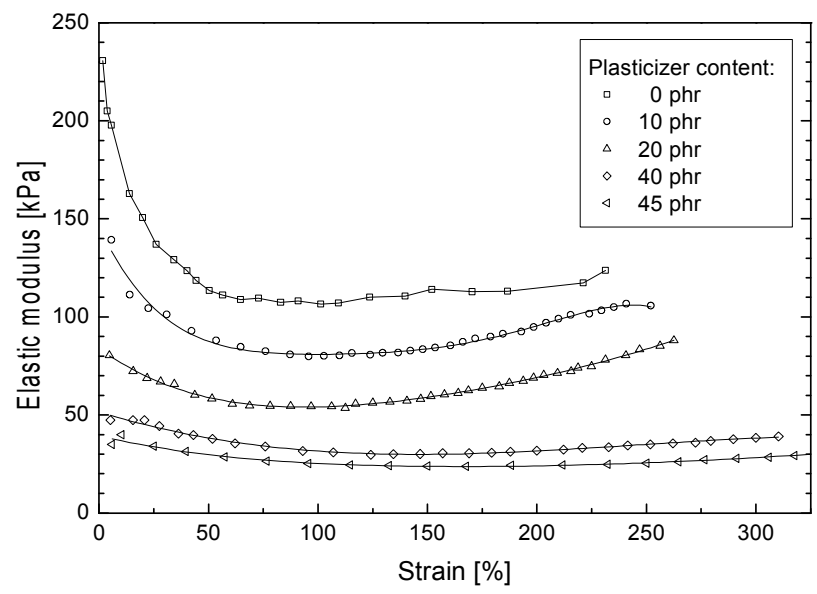

Figure 6. Plots of the tangent elastic modulus at different strains for the elastomers with different content of plasticizer (lines are just guides for the eye).

From DMTA analysis at $100 \mathrm{~Hz}$ (Fig. 7), it is found that all the formulations show two very different mechanical regimes, respectively below and above their glass transition temperatures, which were found to be located in the interval -35 to $-37^{\circ} \mathrm{C}$. At temperatures below the glass transition, the real part of the elastic modulus for all the tested formulations decreases as the amount of plasticizer is increased (Fig. 7a). Although below $T_{g}$ also the imaginary part of the elastic modulus is found to be monotonically reduced in plasticized samples, the dumping ratio of all the formulations, $\tan \delta$, shows a marked change of response across $T_{g}$ : above it, all the formulations become more dissipative, as expected when a system enters a viscoelastic regime (Fig. 7b, inset). However, above $T_{g}$ all the DMTA curves are almost superimposed, so that the viscoelastic properties appear to be less affected by the plasticizer content.

Indeed, by comparing the quasi-static stress-strain behaviour with the data of the DMTA analysis at $100 \mathrm{~Hz}$, we conclude that at room temperature the content of plasticizer mainly affects the mechanical response to slow time-varying stimuli, while it becomes less influencing as the stress-strain variations become faster. 

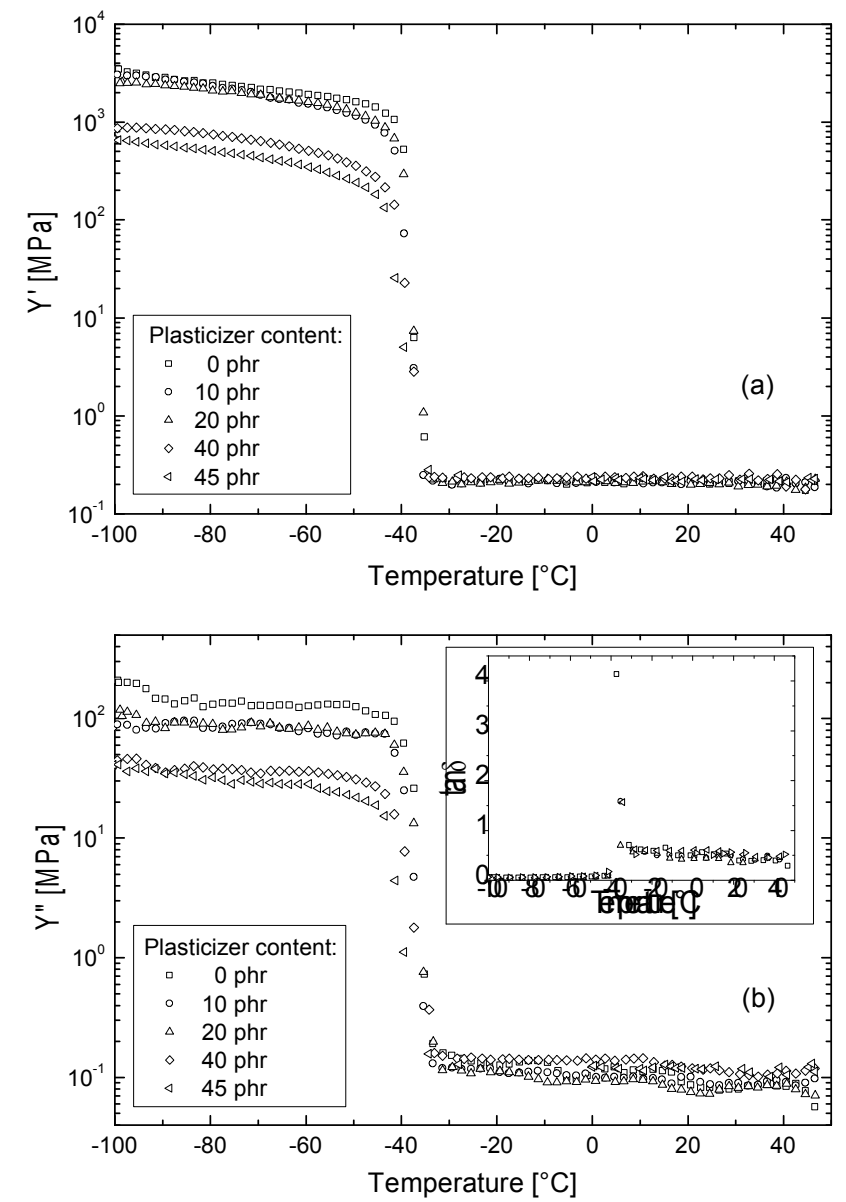

Figure7. DMTA analysis at $100 \mathrm{~Hz}$ of the elastomers at different plasticizer contents: real part (a) and imaginary part (b) of the elastic modulus as a function of temperature.

In order to deepen the mechanical characterization, a DMA analysis of the behaviour of the elastic modulus versus frequency in the range $0.1-100 \mathrm{~Hz}$ was carried out at room temperature for the sample containing $40 \mathrm{phr}$ of plasticizer. Results are reported in Fig. 8. As expected, due to its high softness, the material shows a flat behaviour at low frequencies, up to about $10 \mathrm{~Hz}$. In this range, the elastic modulus $Y^{\prime}$ of the sample remains below $50 \mathrm{kPa}$, consistently with results obtained from the quasi-static elongation test (Fig. 6). However, as the frequency increases over $10 \mathrm{~Hz}$ (Fig. 8a), $Y^{\prime}$ starts to rapidly grow, such that a value of more than $200 \mathrm{kPa}$ is reached at $100 \mathrm{~Hz}$, which is in agreement with the value obtained from the DMTA at $100 \mathrm{~Hz}$ above the glass transition temperature (Fig. 7a). 

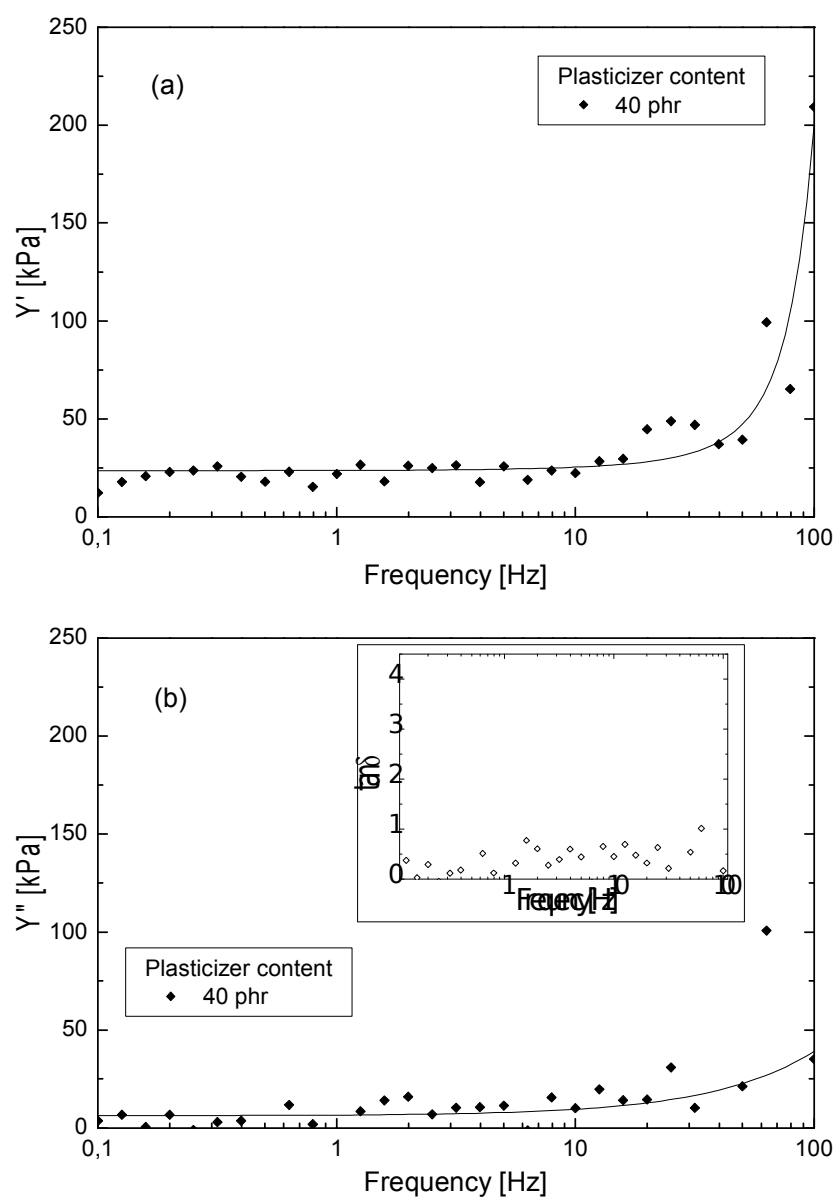

Figure 8. DMA analysis at $22{ }^{\circ} \mathrm{C}$ of the elastomer containing $40 \mathrm{phr}$ of plasticizer. Real part (a) and imaginary part (b) of the elastic modulus as a function of frequency (lines are just guides for the eye).

The assessed decrease of the material's elastic modulus at low frequencies has a straightforwardly direct effect on the achievable active strain in response to an applied electric field. Actually, Fig. 9 shows that a higher content of plasticizer enables a higher actuation, for the same applied field. According to Eq. (1), this dependence should not include substantial electrical effects, since the dielectric permittivity was found to be affected very marginally by the plasticizer content (Fig. 4a). Nevertheless, Fig. 9 also shows that the progressive addition of plasticizer over a $20 \mathrm{phr}$ content caused a progressive decrease of the electrical failure threshold for the material in the tested conditions. Regardless of the origin of the particular breakdown mechanisms that might take place in the different samples, this evidence indicates that, while higher amounts of plasticizer allow for relatively higher strains (i.e. larger strains at the same nominal field), they also reduce the maximum achievable strains in absolute terms. It is thus clear that the proper choice of plasticizer amount must derive from a trade-off between different requirements in terms of performance.

All the curves of the tested silicone samples, except for that of pure matrix without plasticizer, lie upon the characteristic of VHB 4905, indicating that, for the same applied electric field and for the same pre-strain of $100 \%$, this silicone exhibits a higher actuation strain as compared to VHB 4905 (3.1\% for the silicone sample containing $45 \mathrm{phr}$ plasticizer at $7 \mathrm{~V} / \mu \mathrm{m}$ against $0.2 \%$ for the VHB 4905 at the same electric field). On the other hand, it is worth remarking also that the VHB 4905 elastomer may be capable of much higher strains in absolute terms, as it can be driven at electric fields much higher than the reported $15 \mathrm{~V} / \mu \mathrm{m}$. The comparison made here was only aimed at showing the electromechanical response of the two materials in the same conditions. 


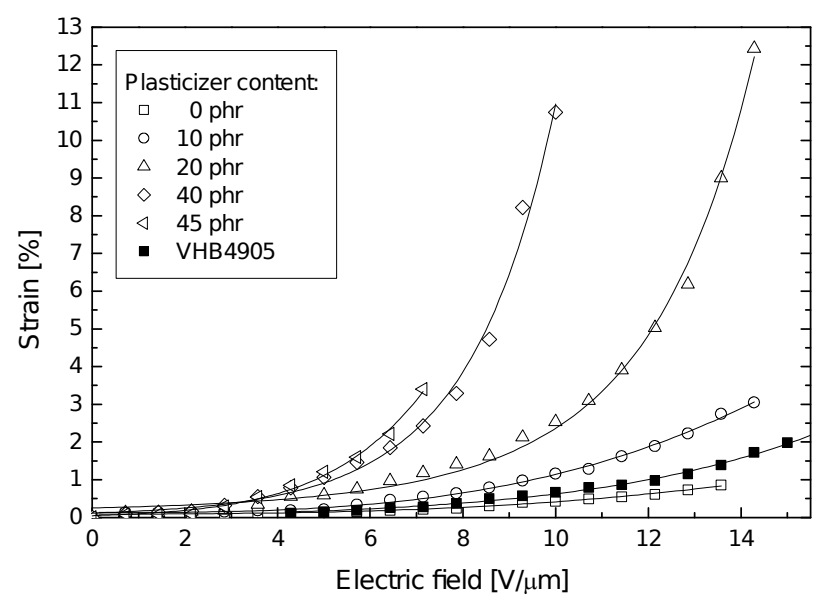

Figure 9. Electromechanical response of the elastomers with different content of plasticizer (lines are just guides for the eye).

\section{Conclusions}

An extensive characterisation of a silicone dielectric elastomer for actuation was presented, and the effects caused by the addition of variable amounts of plasticizer on the properties relevant for actuation purposes were studied. Results elucidated the role played by the plasticiser in several respect. In particular, it came out that for the tested silicone formulations the good performance achieved in the electromechanical tests must be mainly ascribed to their mechanical properties (low elastic modulus) rather than their dielectric response. Indeed, the dielectric constant of such systems was found to be low and, unlike the elastic modulus, it was almost unaffected by plasticization. In any case, it resulted that the increase in the mechanical compliance provided by plasticization had positive impact on the electromechanical response, since it decreased the electric field needed to obtain the same actuation strain level. In this respect, it was also showed that plasticization was able to make the starting silicone to perform better than the widely used VHB 4905 dielectric elastomer. These investigations might offer a useful guide to actuator designers, to adjust the desirable material properties according to specific application needs.

\section{Acknowledgments}

Partial financial support is gratefully acknowledged from COST (European Cooperation in Science and Technology) in the framework of 'ESNAM - European Scientific Network for Artificial Muscles' (COST Action MP1003), from Fondazione Cassa di Risparmio di Pisa in the framework of the project 'POLOPTEL' (no. 167/09), and from the European Commission in the framework of the project 'CEEDS: The Collective Experience of Empathic Data Systems' (FP7-ICT-2009.8.4, Grant 258749).

\section{References}

[1] Carpi F. and Smela E., Editors. 2009 Biomedical applications of electroactive polymer actuators (Chichester: Wiley).

[2] Pelrine R, Kornbluh R, Pei Q and Joseph J 2000 High-speed electrically actuated elastomers with strain greater than $100 \%$ Science $\mathbf{2 8 7} 836-839$

[3] Brochu P and Pei Q 2010 Advances in dielectric elastomers for actuators and artificial muscles Macromol. Rapid Commun. 31 10-36 
[4] Carpi F, De Rossi D, Kornbluh R, Pelrine R and Sommer-Larsen P Editors 2008 Dielectric Elastomers as Electromechanical Transducers. Fundamentals, Materials, Devices, Models \& Applications of an Emerging Electroactive Polymer Technology (Oxford: Elsevier)

[5] Carpi F, Bauer S and De Rossi D 2010 Stretching dielectric elastomer performance Science 330 $1759-1761$

[6] Akbari S, Shea H R. 2012 An array of $100 \mu \mathrm{m} \times 100 \mu \mathrm{m}$ dielectric elastomer actuators with $80 \%$ strain for tissue engineering applications. Sensors and Actuators A 186 236- 241.

[7] Aschwanden M, Beck M, Stemmer A 2007 Diffractive Transmission Grating Tuned by Dielectric Elastomer Actuator Photonics Technology Letters IEEE 19 1090-1092

[8] Carpi F, Frediani G, Turco S, De Rossi D. 2011 Bioinspired Tunable Lens with Muscle-Like Electroactive Elastomers. Advanced Functional Materials 21 4152-4158

[9] Niu X, Stoyanov H, Hu W, Leo R, Brochu P, Pei Q. 2013 Synthesizing a new dielectric elastomer exhibiting large actuation strain and suppressed electromechanical instability without prestretching. Journal of Polymer Science, Part B: Polymer Physics 51 197-206

[10] Li T, Keplinger C, Baumgartner R, Bauer S, Yang W, Suo Z. 2013 Giant voltage-induced deformation in dielectric elastomers near the verge of snap-through instability. Journal of the Mechanics and Physics of Solids 61 611-628

[11] Carpi F, Migliore A, Serra G and De Rossi D 2005 Helical dielectric elastomer actuators Smart Mater. Struct. 14 1210-1216

[12] Carpi F, Salaris C and De Rossi D 2007 Folded dielectric elastomer actuators Smart Mater. Struct. 16 S300-S305.

[13] Carpi F, Fantoni G, Frediani G and De Rossi D 2008 Buckling actuators with integrated displacement sensor, In Carpi F, De Rossi D, Kornbluh R, Pelrine R and Sommer-Larsen P Editors 2008 Dielectric Elastomers as Electromechanical Transducers. Fundamentals, Materials, Devices, Models \& Applications of an Emerging Electroactive Polymer Technology (Oxford: Elsevier).

[14] Carpi F, Frediani G and De Rossi D 2010 Hydrostatically coupled dielectric elastomer actuators. IEEE-ASME Transactions On Mechatronics. 15(2) 308-315.

[15] Carpi F and De Rossi D 2005 Improvement of electromechanical actuating performances of a silicone dielectric elastomer by dispersion of titanium dioxide powder IEEE T. Dielect. El. In. 12 835-843

[16] Gallone G, Carpi F, De Rossi D, Levita G and Marchetti A 2007 Dielectric constant enhancement in a silicone elastomer filled with lead magnesium niobate-lead titanate Mat. Sci. Eng. C 27 110-116

[17] Carpi F, Gallone G, Galantini F and De Rossi D 2008 Silicone-Poly(hexylthiophene) blends as elastomers with enhanced electromechanical transduction properties Adv. Funct. Mater. 18 235-241

[18] Gallone G, Galantini F and Carpi F 2010 Perspectives for new dielectric elastomers with improved electromechanical actuation performance: Composites versus blends Polym. Int. 59 400-406

[19] Pelrine R. E., Kornbluh R. D. and Joseph J. P. 1998 Electrostriction of polymer dielectrics with compliant electrodes as a means of actuation. Sensors and Actuators A-Phys. 64 77-85. 